\title{
ON AN INEQUALITY OF DIANANDA. PART III
}

PENG GAO

Received 30 January 2006; Revised 3 May 2006; Accepted 28 May 2006

We extend the results in parts I, II on certain inequalities involving the generalized power means.

Copyright (c) 2006 Hindawi Publishing Corporation. All rights reserved.

\section{Introduction}

Let $M_{n, r}(\mathbf{x})$ be the generalized weighted means $M_{n, r}(\mathbf{x})=\left(\sum_{i=1}^{n} q_{i} x_{i}^{r}\right)^{1 / r}$, where $M_{n, 0}(\mathbf{x})$ denotes the limit of $M_{n, r}(\mathbf{x})$ as $r \rightarrow 0^{+}, \mathbf{x}=\left(x_{1}, x_{2}, \ldots, x_{n}\right)$, and $q_{i}>0(1 \leq i \leq n)$ are positive real numbers with $\sum_{i=1}^{n} q_{i}=1$. In this paper, we let $q=\min q_{i}$ and always assume $n \geq 2,0 \leq x_{1}<x_{2}<\cdots<x_{n}$.

We define $A_{n}(\mathbf{x})=M_{n, 1}(\mathbf{x}), G_{n}(\mathbf{x})=M_{n, 0}(\mathbf{x}), H_{n}(\mathbf{x})=M_{n,-1}(\mathbf{x})$ and we will write $M_{n, r}$ for $M_{n, r}(\mathbf{x}), A_{n}$ for $A_{n}(\mathbf{x})$, and similarly for other means when there is no risk of confusion.

For real numbers $\alpha, \beta$ and mutually distinct numbers $r, s, t$, we define

$$
\Delta_{r, s, t, \alpha, \beta}=\left|\frac{M_{n, r}^{\alpha}-M_{n, t}^{\alpha}}{M_{n, r}^{\beta}-M_{n, s}^{\beta}}\right|,
$$

where we interpret $M_{n, r}^{0}-M_{n, s}^{0}$ as $\ln M_{n, r}-\ln M_{n, s}$. When $\alpha=\beta$, we define $\Delta_{r, s, t, \alpha}$ to be $\Delta_{r, s, t, \alpha, \alpha}$. We also define $\Delta_{r, s, t}$ to be $\Delta_{r, s, t, 1}$.

For $r>s>t \geq 0, \alpha>0$, we studied in [7,8] inequalities of the following two types:

$$
\begin{gathered}
C_{r, s, t}\left((1-q)^{\alpha}\right) \geq \Delta_{r, s, t, \alpha}, \\
\Delta_{r, s, t, \alpha} \geq C_{r, s, t}\left(q^{\alpha}\right),
\end{gathered}
$$

where

$$
C_{r, s, t}(x)=\frac{1-x^{1 / t-1 / r}}{1-x^{1 / s-1 / r}}, \quad t>0 ; \quad C_{r, s, 0}(x)=\frac{1}{1-x^{1 / s-1 / r}}
$$


For any set $\{a, b, c\}$ with $a, b, c$ mutually distinct and nonnegative, we let $r=\max \{a$, $b, c\}, t=\min \{a, b, c\}, s=\{a, b, c\} \backslash\{r, t\}$. By saying (1.2) (resp., (1.3)) holds for the set $\{a, b, c\}, \alpha>0$, we mean (1.2) (resp., (1.3)) holds for $r>s>t \geq 0, \alpha>0$. The main result in $[8]$ is the following.

Theorem 1.1. Inequality (1.2) holds for the set $\{r, s, 1\}$ with $\alpha=1, r, s, 1$ mutually distinct and $r>s \geq 0, r+s \geq 1$. The equality holds if and only if $n=2, x_{1}=0, q_{1}=q$.

The consideration of $n=2, x_{2} \rightarrow x_{1}$ shows that inequalities (1.2) and (1.3) cannot hold simultaneously in general. By [8, Lemma 2.1], $C_{r, s, t}(x)$ is an increasing function of $x$ for $0<x<1$. Hence in order for (1.2) to hold, it is necessary to have $C_{r, s, t}\left((1 / 2)^{\alpha}\right) \geq$ $(r-t) /(r-s)$. In this paper, we will complete our discussion on (1.2) for the case $\alpha=1$ and $1 \in\{r, s, t\}$. We will also show that for $t>0$, inequality (1.3) does not hold.

As an analogue of (1.2), we note the following result of $\mathrm{Wu}[16]$.

Theorem 1.2 [16, Theorem 2]. For $x_{i}>0,1 \leq i \leq n, \lambda \geq \max \left\{(n-1)^{p-1},(p-1)^{p-1}\right\}$, $p>1$,

$$
\left(\sum_{i=1}^{n} x_{i}\right)^{p} \leq \lambda \sum_{i=1}^{n} x_{i}^{p}+\left(n^{p}-n \lambda\right)\left(\prod_{i=1}^{n} x_{i}\right)^{p / n} .
$$

The case $1<p \leq n$ in the above theorem is of particular interest to us and we will give another proof of this case in Section 3. We will also use the idea in Wu's proof of Theorem 1.2 to obtain results of similar kinds.

The one-parameter mean value family

$$
L_{r}=L_{r}(x, y)=\left(\frac{x^{r}-y^{r}}{r(x-y)}\right)^{1 /(r-1)} \quad(r \neq 0,1, x, y>0, x \neq y)
$$

is known as Stolarsky's generalized logarithmic mean [14]. We note here the limit relations

$$
\begin{aligned}
& \lim _{r \rightarrow 0} L_{r}(x, y)=L(x, y)=\frac{x-y}{\log x-\log y} ; \\
& \lim _{r \rightarrow 1} L_{r}(x, y)=I(x, y)=\frac{1}{e}\left(\frac{x^{x}}{y^{y}}\right)^{1 /(x-y)}
\end{aligned}
$$

are called the logarithmic and identric means, respectively. We refer the reader to paper [2] and the references therein for many inequalities involving $L, I$, and the generalized power means. Some of these inequalities can be regarded as analogues of inequalities (1.2) and (1.3) considered here. We will derive several inequalities involving the $L_{r} s$ by applying (1.2) and (1.3) in the last part of this paper.

\section{An extension of Theorem 1.1}

Theorem 2.1. Let $1>r>s \geq 0$. If $C_{1, r, s}(1 / 2) \geq(1-s) /(1-r)$, then inequality (1.2) holds for the set $\{1, r, s\}$ with $\alpha=1$. 
Proof. The case $s=0$ was treated in [7] and the case $r+s \geq 1$ was treated in [8], so we may assume $s>0$ and $r+s<1$ from now on. Define

$$
D_{n}(\mathbf{x})=A_{n}-M_{n, r}-C(1-q)\left(A_{n}-M_{n, s}\right), \quad C(x)=\frac{1-x^{1 / r-1}}{1-x^{1 / s-1}} .
$$

We need to show $D_{n} \geq 0$ and we have

$$
\frac{1}{q_{n}} \frac{\partial D_{n}}{\partial x_{n}}=1-M_{n, r}^{1-r} x_{n}^{r-1}-C(1-q)\left(1-M_{n, s}^{1-s} x_{n}^{s-1}\right) .
$$

By a change of variables $x_{i} / x_{n} \rightarrow x_{i}, 1 \leq i \leq n$, we may assume $0 \leq x_{1}<x_{2}<\cdots<x_{n}=1$ in (2.2) and rewrite it as

$$
g_{n}\left(x_{1}, \ldots, x_{n-1}\right):=1-M_{n, r}^{1-r}-C(1-q)\left(1-M_{n, s}^{1-s}\right) .
$$

We want to show $g_{n} \geq 0$. Let $\mathbf{a}=\left(a_{1}, \ldots, a_{n-1}\right) \in[0,1]^{n-1}$ be the point in which the absolute minimum of $g_{n}$ is reached. We may assume $a_{1} \leq a_{2} \leq \cdots \leq a_{n-1}$. If $a_{i}=a_{i+1}$ for some $1 \leq i \leq n-2$ or $a_{n-1}=1$, by combining $a_{i}$ with $a_{i+1}$ and $q_{i}$ with $q_{i+1}$ or $a_{n-1}$ with 1 and $q_{n-1}$ with $q_{n}$, it follows from [8, Lemma 2.1] that we can reduce the determination of the absolute minimum of $g_{n}$ to that of $g_{n-1}$ with different weights. Thus without loss of generality, we may assume $a_{1}<a_{2}<\cdots<a_{n-1}<1$.

If $\mathbf{a}$ is a boundary point of $[0,1]^{n-1}$, then $a_{1}=0$, and we can regard $g_{n}$ as a function of $a_{2}, \ldots, a_{n-1}$, then we obtain

$$
\nabla g_{n}\left(a_{2}, \ldots, a_{n-1}\right)=0
$$

Otherwise, $a_{1}>0$, $\mathbf{a}$ is an interior point of $[0,1]^{n-1}$, and

$$
\nabla g_{n}\left(a_{1}, \ldots, a_{n-1}\right)=0 .
$$

In either case, $a_{2}, \ldots, a_{n-1}$ solve the equation

$$
(r-1) M_{n, r}^{1-2 r} x^{r-1}+C(1-q)(1-s) M_{n, s}^{1-2 s} x^{s-1}=0 .
$$

The above equation has at most one root (regarding $M_{n, r}, M_{n, s}$ as constants), so we only need to show $g_{n} \geq 0$ for the case $n=3$ with $0=a_{1}<a_{2}=x<a_{3}=1$ in (2.3). In this case, we regard $g_{3}$ as a function of $x$ and we get

$$
\frac{1}{q_{2}} g_{3}^{\prime}(x)=M_{3, r}^{1-2 r} x^{r-1} h(x),
$$

where

$$
h(x)=r-1+(1-s) C(1-q)\left(q_{2} x^{s / 2}+q_{3} x^{-s / 2}\right)^{(1-2 s) / s}\left(q_{2} x^{r / 2}+q_{3} x^{-r / 2}\right)^{(2 r-1) / r} .
$$

If $q_{2}=0$ (note that $\left.q_{3}>0\right)$, then

$$
h(x)=r-1+(1-s) C(1-q) q_{3}^{1 / s-1 / r} x^{s-r} .
$$


One easily checks that in this case, $h(x)$ has exactly one root in $(0,1)$. Now assume $q_{2}>0$, then

$$
h^{\prime}(x)=(1-s) C(1-q) M_{3, s}^{1-3 s} M_{3, r}^{r-1} x^{-(r+s+2) / 2} p(x),
$$

where

$$
p(x)=(r-s)\left(q_{2}^{2} x^{r+s}-q_{3}^{2}\right)+(r+s-1) q_{2} q_{3}\left(x^{r}-x^{s}\right) .
$$

Now

$$
p^{\prime}(x)=x^{r+s-1}\left(\left(r^{2}-s^{2}\right) q_{2}^{2}+(r+s-1) q_{2} q_{3}\left(r x^{-s}-s x^{-r}\right)\right):=x^{r+s-1} q(x) .
$$

It is easy to see that the only positive root for $q^{\prime}(x)=0$ is $x=1$. Thus $p^{\prime}(x)=0$ can have at most one root in $(0,1)$. This means that $p(x)=0$ has at most two roots in $(0,1)$. Now if $q_{2}>q_{3}$, then it follows from this and that $p(0)<0, p(1)>0$ that $p(x)=0$ hence $h^{\prime}(x)=0$ has only one root in $(0,1)$. Thus $h(x)=0$ can have at most two positive roots in $(0,1]$. Note that $\lim _{x \rightarrow 0^{+}} h(x)=+\infty$ and by our assumption, note that $h(1) \leq 0$. This means that $h(x)$ has at most one root in $(0,1)$ and hence $g_{3}^{\prime}(x)$ has at most one root $x_{0}$ in $(0,1)$. Since $\lim _{x \rightarrow 0^{+}} g_{3}^{\prime}(x)=+\infty, g_{3}(x)$ takes its maximum value at $x_{0}$ and we conclude that $g_{3}(x) \geq \min \left\{g_{3}(0), g_{3}(1)\right\}=0$.

Suppose now $q_{2} \leq q_{3}$. By setting $y=q_{3} / q_{2}$, we can rewrite $p(x)$ as

$$
p(x)=q_{2}^{2}\left((r-s)\left(x^{r+s}-y^{2}\right)+(r+s-1) y\left(x^{r}-x^{s}\right)\right):=q_{2}^{2} f(y) .
$$

We now want to show $f(y) \leq 0$ for $y \geq 1,0 \leq x \leq 1$. We may assume $x>0$ and note that for fixed $x, f(y)$ is a quadratic function with $f(0)>0$. Since the coefficient of $y^{2}$ is negative, it thus suffices to show that $f(1) \leq 0$. Equivalently, we need to show $p(x) \leq 0$ when $q_{2}=q_{3}$ and by repeating the argument in the preceding paragraph, we see that $p^{\prime}(x)=0$ has no root in $(0,1)$, hence $p(x) \leq \max \{p(0), p(1)\} \leq 0$. Thus in this case, $h(x)$ is a decreasing function of $x$ for $0 \leq x \leq 1$. It follows from this that $h(x)=0$ has only one root in $(0,1]$ and similar to the argument in the preceding paragraph, we have $g_{3}(x) \geq$ $\min \left\{g_{3}(0), g_{3}(1)\right\}=0$.

Thus we have shown $g_{n} \geq 0$, hence $\partial D_{n} / \partial x_{n} \geq 0$ with equality holding if and only if $n=1$ or $n=2, x_{1}=0, q_{1}=q$. By letting $x_{n}$ tend to $x_{n-1}$, we have $D_{n} \geq D_{n-1}$ (with weights $\left.q_{1}, \ldots, q_{n-2}, q_{n-1}+q_{n}\right)$. Since $C(1-q)$ is an increasing function of $q$ by [8, Lemma 2.1], it follows by induction that $D_{n}>D_{n-1}>\cdots>D_{2}=0$ when $x_{1}=0, q_{1}=q$ in $D_{2}$. Else, $D_{n}>D_{n-1}>\cdots>D_{1}=0$ and this completes the proof.

Now we show that inequality (1.3) does not hold for $t>0$. It suffices to consider the case $n=2$ with $x_{2}=1, q_{2}=q$. We set $x_{1}=x$ and define

$$
f(x)=M_{2, r}^{\alpha}-M_{2, t}^{\alpha}-C_{r, s, t}\left(q^{\alpha}\right)\left(M_{2, r}^{\alpha}-M_{2, s}^{\alpha}\right) .
$$


Note that $f(0)=0$ and

$$
\frac{f^{\prime}(x)}{\alpha(1-q) x^{t-1}}=M_{2, r}^{\alpha-r} x^{r-t}-M_{2, t}^{\alpha-t}-C_{r, s, t}\left(q^{\alpha}\right)\left(M_{2, r}^{\alpha-r} x^{r-t}-M_{2, s}^{\alpha-s} x^{s-t}\right)
$$

If $t>0$, the right-hand side above is negative when $x=0$, which implies $f(x)<0$ for positive $x$ small enough so that (1.3) fails to hold.

In [8], the author asked whether it is true or not that if (1.2) holds for $r>s>t \geq 0$, $\alpha>0$, then it also holds for $r>s>t \geq 0, k \alpha$ with $0<k<1$, and if (1.3) holds for $r>s>$ $t \geq 0, \alpha>0$, then it also holds for $r>s>t \geq 0, k \alpha$ with $k>1$. Now the second part above is true due to the discussion above since (1.3) only holds when $t=0$ and this case has already been discussed in [8, Theorem 3.2]. We would also like to take the opportunity to correct some typos in the statement of [8, Theorem 3.2], namely, one needs to exchange the two conditions $k>1$ and $0<k<1$ there. We now proceed to show that the first part is actually not true.

We write for convenience that

$$
C=C_{r, s, t}\left((1-q)^{\alpha}\right), \quad C^{\prime}=C_{r, s, t}\left((1-q)^{k \alpha}\right),
$$

so that we can rewrite (1.2) as

$$
M_{n, s}^{\alpha} \leq\left(1-\frac{1}{C}\right) M_{n, r}^{\alpha}+\frac{1}{C} M_{n, t}^{\alpha}
$$

Now, what we assert is that it follows from above that for $0<k<1$,

$$
M_{n, s}^{k \alpha} \leq\left(1-\frac{1}{C^{\prime}}\right) M_{n, r}^{k \alpha}+\frac{1}{C^{\prime}} M_{n, t}^{k \alpha}
$$

Thus to show that the assertion does not hold, it suffices to find an example such that

$$
\left(\left(1-\frac{1}{C}\right) M_{n, r}^{\alpha}+\frac{1}{C} M_{n, t}^{\alpha}\right)^{k}>\left(1-\frac{1}{C^{\prime}}\right) M_{n, r}^{k \alpha}+\frac{1}{C^{\prime}} M_{n, t}^{k \alpha}
$$

Now we set $y=M_{n, r}^{\alpha} / M_{n, t}^{\alpha}$ and note that in the case $n=2, x_{1}=0, q_{2}=q, y=q^{\alpha(1 / r-1 / t)}$. Thus it suffices to show that for $y=q^{\alpha(1 / r-1 / t)}$,

$$
\left(\left(1-\frac{1}{C}\right) y+\frac{1}{C}\right)^{k}>\left(1-\frac{1}{C^{\prime}}\right) y^{k}+\frac{1}{C^{\prime}} .
$$

Let

$$
f(y)=\frac{\left(1-\left(1 / C^{\prime}\right)\right) y^{k}+1 / C^{\prime}}{((1-(1 / C)) y+1 / C)^{k}}
$$


and we want to show $f\left(q^{\alpha(1 / r-1 / t)}\right)<1$. Using the definitions for $C, C^{\prime}$ and the notations

$$
\begin{gathered}
\beta=\alpha\left(\frac{1}{t}-\frac{1}{r}\right), \quad \gamma=\frac{1 / s-1 / r}{1 / t-1 / r}, \quad A=\frac{q^{\beta}-1}{1-(1-q)^{\beta}}, \\
A^{\prime}=\frac{q^{k \beta}-1}{1-(1-q)^{k \beta}}, \quad z=(1-q)^{\beta \gamma},
\end{gathered}
$$

we obtain

$$
f\left(q^{\alpha(1 / r-1 / t)}\right)=\frac{1+A^{\prime}\left(1-z^{k}\right)}{(1+A(1-z))^{k}}:=g(z) .
$$

We note first here that $A, A^{\prime} \leq-1$ and $0 \leq \gamma \leq 1$. It follows that $(1-q)^{\beta} \leq z \leq 1$. Now

$$
g^{\prime}(z)=\frac{k\left(-A^{\prime}(1+A) z^{k-1}+A\left(1+A^{\prime}\right)\right)}{(1+A(1-z))^{k+1}} .
$$

The function

$$
z \longmapsto-A^{\prime}(1+A) z^{k-1}+A\left(1+A^{\prime}\right)
$$

only has one positive root and is $<0$ when $z \rightarrow 0^{+}$as long as $q \neq 1 / 2$. It follows from this and $g\left((1-q)^{\beta}\right)=g(1)=1$ that $g(z)<1$ for $(1-q)^{\beta}<z<1$ if $q \neq 1 / 2$, which is just what is desired.

\section{Another look at Theorem 1.2}

Let $k \in\{0,1, \ldots, n\}$, the $k$ th symmetric function $E_{n, k}$ of $\mathbf{x}$ and its mean $P_{n, k}$ are defined by

$$
E_{n, k}(\mathbf{x})=\sum_{1 \leq i_{1}<\cdots<i_{k} \leq n} \prod_{j=1}^{k} x_{i_{j}}, \quad 1 \leq k \leq n ; \quad E_{n, 0}=1 ; \quad P_{n, k}^{k}(\mathbf{x})=\frac{E_{n, k}(\mathbf{x})}{\left(\begin{array}{l}
n \\
k
\end{array}\right)} .
$$

The following lemma is due to $\mathrm{Wu}$ et al. [17] (see also [1, pages 317-318]).

Lemma 3.1. Let $2 \leq k \leq n, \mathbf{x}=\left(x_{1}, \ldots, x_{n}\right), x_{1} \leq x_{2} \leq \cdots \leq x_{n}$. There exists $\mathbf{y}=\left(y_{1}, \ldots, y_{k}\right)$ with $x_{1} \leq y_{1} \leq \cdots \leq y_{k} \leq x_{n}$ such that $P_{n, i}(\mathbf{x})=P_{k, i}(\mathbf{y}), 0 \leq i \leq k$. Moreover, if $x_{1}, \ldots, x_{n}$ are not all equal, then $y_{1}, \ldots, y_{k}$ are also not all equal.

In this section, we would like to first give another proof of Theorem 1.2 for the case $1<p \leq n$ and we will need a result of Shen [13, Lemma 1], which we will state as follows.

Lemma 3.2. Let $0 \leq x_{i} \leq 1$, then for $1 \leq k \leq n$,

$$
P_{n, k-1}^{k-1}(\mathbf{x}) \leq \frac{1}{k}+\frac{k-1}{k} P_{n, k}^{k}(\mathbf{x}) .
$$

Proof. By Lemma 3.1, there exists $\mathbf{y}=\left(y_{1}, \ldots, y_{k}\right)$ with $0 \leq x_{1} \leq y_{1} \leq \cdots \leq y_{k} \leq x_{n}$ such that $P_{n, k-1}(\mathbf{x})=P_{k, k-1}(\mathbf{y})$ and $P_{n, k}(\mathbf{x})=P_{k, k}(\mathbf{y})$. It thus suffices to prove the lemma for the case $n=k$ and one checks easily that this follows from case (ii) of [13, Lemma 1]. 
One can then easily deduce from above the original result of Shen [13, Lemma 1] which we will need, namely, for $0 \leq x_{i} \leq 1$,

$$
P_{n, 1}(\mathbf{x}) \leq \frac{n-1}{n}+\frac{1}{n} P_{n, n}^{n}(\mathbf{x})
$$

We now use Lemma 3.2 to deduce the following.

Corollary 3.3. For $1<p \leq n$,

$$
\left(\sum_{i=1}^{n} x_{i}\right)^{p} \leq(n-1)^{p-1} \sum_{i=1}^{n} x_{i}^{p}+n\left(n^{p-1}-(n-1)^{p-1}\right)\left(\prod_{i=1}^{n} x_{i}\right)^{p / n} .
$$

Proof. In this proof, we assume that $0 \leq x_{1} \leq \cdots \leq x_{n}$ and we define

$$
f(\mathbf{x})=(n-1)^{p-1} \sum_{i=1}^{n} x_{i}^{p}+n\left(n^{p-1}-(n-1)^{p-1}\right)\left(\prod_{i=1}^{n} x_{i}\right)^{p / n}-\left(\sum_{i=1}^{n} x_{i}\right)^{p} .
$$

If $x_{n}=0$ or $x_{1}=\cdots=x_{n}$, then $f=0$, otherwise, we may assume $n \geq 2$ and $x_{k-1}<x_{k}=$ $\cdots=x_{n}=x$ for some $1 \leq k \leq n$ (we set $x_{0}=0$ here), then

$$
\frac{1}{(n-k+1) p x^{p-1}} \frac{\partial f}{\partial x}=(n-1)^{p-1}+\left(n^{p-1}-(n-1)^{p-1}\right)\left(\prod_{i=1}^{n} \frac{x_{i}}{x}\right)^{p / n}-\left(\sum_{i=1}^{n} \frac{x_{i}}{x}\right)^{p-1}
$$

We want to show that the right-hand side above is nonnegative. Note that $0 \leq x_{i} / x \leq 1$, hence by a change of variables $x_{i} / x \rightarrow x_{i}$, it suffices to show that

$$
(n-1)^{p-1}+\left(n^{p-1}-(n-1)^{p-1}\right)\left(\prod_{i=1}^{n} x_{i}\right)^{p / n}-\left(\sum_{i=1}^{n} x_{i}\right)^{p-1} \geq 0
$$

for $0 \leq x_{i} \leq 1$ and $1<p \leq n$. By (3.3), we only need to show that

$$
\left(n-1+\prod_{i=1}^{n} x_{i}\right)^{p-1} \leq(n-1)^{p-1}+\left(n^{p-1}-(n-1)^{p-1}\right)\left(\prod_{i=1}^{n} x_{i}\right)^{p / n} .
$$

By further setting

$$
t=\left(\prod_{i=1}^{n} x_{i}\right)^{p / n}
$$

it suffices to show that

$$
g(t)=(n-1)^{p-1}+\left(n^{p-1}-(n-1)^{p-1}\right) t-\left(n-1+t^{n / p}\right)^{p-1} \geq 0
$$


for $0 \leq t \leq 1$. One checks easily that

$$
g^{\prime \prime}(t)=-\frac{n(p-1)}{p}\left(n-1+t^{n / p}\right)^{p-3} t^{n / p-2} h(t),
$$

where

$$
\begin{aligned}
h(t) & =\left(n-\frac{n}{p}-1\right) t^{n / p}+\left(\frac{n}{p}-1\right)(n-1) \geq \min \{h(0), h(1)\} \\
& =\min \left\{\frac{n}{p}(n-2),\left(\frac{n}{p}-1\right)(n-1)\right\} \geq 0 .
\end{aligned}
$$

We conclude that $g(t)$ is a concave function for $0 \leq t \leq 1$. Hence

$$
g(t) \geq \min \{g(0), g(1)\}=0 .
$$

It follows from this that $\partial f / \partial x \geq 0$ and by letting $x \rightarrow x_{k-1}$ and repeating the above argument, we conclude that $f(\mathbf{x}) \geq f\left(x_{1}, x_{1}, \ldots, x_{1}\right)=0$ which completes the proof.

We note here that it is pointed out in [9] that Corollary 3.3 was obtained by Chen and Wang [6], however, we have no access to the paper for the method used in their proof. We also note that by a change of variables $x_{i} \rightarrow x_{i}^{1 / p},(3.4)$ is equivalent to the case $r=1$, $s=1 / p, t=0, \alpha=1$ of (1.2) and this certainly improves [7, Theorem 3.1].

Similarly, in another paper of Chen and Wang [5], they have shown that for $n \geq 2$, $p \geq n /(n-1)$,

$$
\left(\sum_{i=1}^{n} x_{i}\right)^{p} \geq \sum_{i=1}^{n} x_{i}^{p}+\left(n^{p}-n\right)\left(\prod_{i=1}^{n} x_{i}\right)^{p / n} .
$$

Again, by a change of variables $x_{i} \rightarrow x_{i}^{1 / p}$, this is equivalent to the case $r=1, s=1 / p, t=0$, $\alpha=1$ of (1.3), which also improves [7, Theorem 3.1].

In [9], the following result was established.

Theorem 3.4. Let $q_{i}=1 / n$, then for any integer $2 \leq p \leq n$,

$$
\left(\sum_{i=1}^{n} x_{i}\right)^{p} \leq\left(n^{p}-\lambda\left(\begin{array}{l}
n \\
p
\end{array}\right)\right) M_{n, 2}^{p}+\lambda E_{n, p},
$$

with

$$
\lambda=\frac{n^{p}(1-1 / n)^{p / 2}-(n-1)^{p}}{\left(\begin{array}{c}
n \\
p
\end{array}\right)(1-1 / n)^{p / 2}-\left(\begin{array}{c}
n-1 \\
p
\end{array}\right)} .
$$

To the author's knowledge, the proof given in [9] for the above theorem is not quite correct and we will treat more of the relevant topics in a forthcoming paper. We now use a method in [16] (see the proof of Theorem 2 there) to prove a result similar to the above one. We note also that the same method can be applied to give another proof of [7, Theorem 3.1] as well as (3.14) and we will leave the details to the interested reader. 
Peng Gao 9

Theorem 3.5. For $r \geq 2,0<p \leq n$,

$$
\left(\sum_{i=1}^{n} x_{i}\right)^{p} \leq(n-1)^{p(r-1) / r}\left(\sum_{i=1}^{n} x_{i}^{r}\right)^{p / r}+\left(n^{p}-n^{p / r}(n-1)^{p(r-1) / r}\right)\left(\prod_{i=1}^{n} x_{i}\right)^{p / n} .
$$

For $1<r \leq 2, p \geq n /(n-1)$,

$$
\left(\sum_{i=1}^{n} x_{i}\right)^{p} \geq\left(\sum_{i=1}^{n} x_{i}^{r}\right)^{p / r}+\left(n^{p}-n^{p / r}\right)\left(\prod_{i=1}^{n} x_{i}\right)^{p / n}
$$

Proof. We first note that by a change of variables $x_{i} \rightarrow x_{i}^{1 / r},(3.17)$ is equivalent to (1.2) for the set $\{1,1 / r, 0\}$ with $\alpha=p / r$ and (3.18) is equivalent to (1.3) for the set $\{1,1 / r, 0\}$ with $\alpha=p / r$. Thus by [8, Theorem 3.2], it suffices to prove (3.17) for the case $p=n$ and (3.18) for the case $p=n /(n-1)$.

We first prove (3.17) and we consider the quotient

$$
f(\mathbf{x}):=\frac{\left(\sum_{i=1}^{n} x_{i}\right)^{n}-(n-1)^{n(r-1) / r}\left(\sum_{i=1}^{n} x_{i}^{r}\right)^{n / r}}{\prod_{i=1}^{n} x_{i}} .
$$

As in the proof of Corollary 3.3, it suffices to consider the situation $0<x=x_{1}=\cdots=$ $x_{k}<x_{k+1}$ for some $1 \leq k<n$ and to show that in this case, $\partial f / \partial x>0$. Without loss of generality, we assume from now on that $x_{1}<x_{2}$. By setting

$$
t=\left(\frac{\sum_{i=2}^{n} x_{i}^{r}}{n-1}\right)^{1 / r}
$$

we obtain

$$
\begin{aligned}
& x_{1}\left(\prod_{i=1}^{n} x_{i}\right) \frac{\partial f}{(n-1) \partial x_{1}} \\
& \quad=\left(\sum_{i=1}^{n} x_{i}\right)^{n-1}\left(x_{1}-\frac{\sum_{i=2}^{n} x_{i}}{n-1}\right)-(n-1)^{n(r-1) / r}\left(\sum_{i=1}^{n} x_{i}^{r}\right)^{n / r-1}\left(x_{1}^{r}-t^{r}\right) \\
& \quad \geq(n-1)^{n-1}\left(\left(\frac{x_{1}}{n-1}+\frac{\sum_{i=2}^{n} x_{i}}{n-1}\right)^{n-1}\left(x_{1}-t\right)-\left(\frac{x_{1}^{r}}{n-1}+t^{r}\right)^{n / r-1}\left(x_{1}^{r}-t^{r}\right)\right) \\
& \quad \geq(n-1)^{n-1}\left(\left(\frac{x_{1}}{n-1}+t\right)^{n-1}\left(x_{1}-t\right)-\left(\frac{x_{1}^{r}}{n-1}+t^{r}\right)^{n / r-1}\left(x_{1}^{r}-t^{r}\right)\right) \\
& \quad=(n-1)^{n-1} x_{1}^{n}\left(\left(\frac{1}{n-1}+\frac{t}{x_{1}}\right)^{n-1}\left(1-\frac{t}{x_{1}}\right)-\left(\frac{1}{n-1}+\left(\frac{t}{x_{1}}\right)^{r}\right)^{n / r-1}\left(1-\left(\frac{t}{x_{1}}\right)^{r}\right)\right) .
\end{aligned}
$$


We want to show that the last expression above is positive and on setting $y=t x_{1}$, this is equivalent to showing that

$$
g(y, n)=\frac{(1 /(n-1)+y)^{n-1}(y-1)}{\left(1 /(n-1)+y^{r}\right)^{n / r-1}\left(y^{r}-1\right)} \leq 1
$$

for $y \geq 1$. Calculation yields

$$
\begin{aligned}
& \frac{\left(\left(1 /(n-1)+y^{r}\right)^{n / r-1}\left(y^{r}-1\right)\right)^{2}}{(1 /(n-1)+y)^{n-2}\left(1 /(n-1)+y^{r}\right)^{n / r-2}} \frac{\partial g}{\partial y} \\
& =\left(n y+\frac{1}{n-1}-(n-1)\right)\left(y^{r}-1\right)\left(y^{r}+\frac{1}{n-1}\right) \\
& \quad-\left(y+\frac{1}{n-1}\right)\left(y^{r}-y^{r-1}\right)\left(n y^{r}+\frac{r}{n-1}-(n-r)\right) \\
& =\frac{n}{n-1}\left(y^{2 r-1}+(1-r) y^{r+1}+\frac{n-2}{n-1}(r-1) y^{r}+\left(\frac{r}{n-1}-1\right) y^{r-1}-y+\frac{n-2}{n-1}\right) .
\end{aligned}
$$

We now set $s=1 /(n-1)$ so that $0 \leq s \leq 1$ and we define

$$
a(y, s)=y^{2 r-1}+(1-r) y^{r+1}+(1-s)(r-1) y^{r}+(r s-1) y^{r-1}-y+(1-s) .
$$

It then follows from Cauchy's mean value theorem that

$$
\frac{\partial a}{\partial s}=-(r-1) y^{r}+r y^{r-1}-1=r y^{r-1}(1-y)-\left(1-y^{r}\right) \leq 0
$$

Thus

$$
a(y, s) \geq a(y, 1)=y\left(y^{2 r-2}+(1-r) y^{r}+(r-1) y^{r-2}-1\right):=y \cdot b(y) .
$$

Now

$$
b^{\prime}(y)=(r-1) y^{r-3}\left(2 y^{r}-r y^{2}+r-2\right):=(r-1) y^{r-3} c(y) .
$$

One checks easily that for $r \geq 2$, the function $c(y)$ is an increasing function of $y \geq 1$ and hence $c(y) \geq c(1)=0$ so that $b(y)$ is an increasing function of $y \geq 1$ and that $b(y) \geq$ $b(1)=0$. This implies that $a(y, s) \geq 0$ so that $g(y, n)$ is an increasing function of $y$ and we then deduce that

$$
g(y, n) \leq \lim _{y \rightarrow+\infty} g(y, n)=1 .
$$

This shows that $\partial f / \partial x_{1} \geq 0$ and (3.17) now follows from our discussions above.

Now, to prove (3.18), we consider

$$
h(\mathbf{x}):=\frac{\left(\sum_{i=1}^{n} x_{i}\right)^{n /(n-1)}-\left(\sum_{i=1}^{n} x_{i}^{r}\right)^{n /(n-1) r}}{\left(\prod_{i=1}^{n} x_{i}\right)^{1 /(n-1)}} .
$$


Similar to our discussion above, one can assume $x_{n-1}<x_{n}$ and it suffices to show $\partial h / \partial x_{n}>$ 0 . Note that

$$
\begin{aligned}
x_{n}\left(\prod_{i=1}^{n} x_{i}\right)^{1 /(n-1)} \frac{\partial h}{\partial x_{n}}= & (n-1)^{1 /(n-1)}\left(\frac{x_{n}}{n-1}+\frac{\sum_{i=1}^{n-1} x_{i}}{n-1}\right)^{1 /(n-1)}\left(x_{n}-\frac{\sum_{i=1}^{n-1} x_{i}}{n-1}\right) \\
& -\left(\sum_{i=1}^{n} x_{i}^{r}\right)^{n /(n-1) r-1}\left(x_{n}^{r}-\frac{\sum_{i=1}^{n-1} x_{i}^{r}}{n-1}\right) \\
\geq & (n-1)^{1 /(n-1)}\left(\frac{x_{n}}{n-1}+\left(\frac{\sum_{i=1}^{n-1} x_{i}}{n-1}\right)^{1 / r}\right)^{1 /(n-1)}\left(x_{n}-\left(\frac{\sum_{i=1}^{n-1} x_{i}}{n-1}\right)^{1 / r}\right) \\
& -\left(\sum_{i=1}^{n} x_{i}^{r}\right)^{n /(n-1) r-1}\left(x_{n}^{r}-\frac{\sum_{i=1}^{n-1} x_{i}^{r}}{n-1}\right),
\end{aligned}
$$

where the inequality follows from the observation that the function

$$
z \longmapsto\left(\frac{x_{n}}{n-1}+z\right)^{1 /(n-1)}\left(x_{n}-z\right)
$$

is decreasing for $0<z<x_{n}$.

By proceeding similarly as in the proof of (3.17) above, one is then able to establish (3.18) and we will omit all the details here.

We point out here that inequality (3.17) does not hold for $p>n$. To see this, we consider the case $x_{1}=t, x_{2}=\cdots=x_{n}=1$, in which case (3.17) is reduced to

$$
(n-1)^{p(r-1) / r}\left(n-1+t^{r}\right)^{p / r}+\left(n^{p}-n^{p / r}(n-1)^{p(r-1) / r}\right) t^{p / n}-(t+n-1)^{p} \geq 0 .
$$

We denote the left-hand side above as $f(t)$, then one checks easily that $f^{\prime}(0)<0$ for $p>n$ and $f(0)=0$. This means that $f(t)<0$ for $t>0$ sufficiently small and consequently, (3.17) does not hold in this case. Similarly, the case $x_{1}=x_{2}=\cdots=x_{n-1}=t, x_{n}=1$ shows that (3.18) does not hold for $p<n /(n-1)$.

\section{Inequalities involving the generalized logarithmic mean}

In this section, the mean $M_{2, r}$ is always equipped with $q_{1}=q_{2}=1 / 2$ and in this case, we note that $L_{2}=A_{2}$ and $L_{-1}=G_{2}$. Now we need a result of Pittenger [10].

Theorem 4.1. Let $a_{1}(r)=(r+1) / 3$ and let $a_{2}(r)=(r-1) \log 2 / \log r$ for $r>0$ with $a_{2}(1)=$ $\log$ 2. For $r>0$, let $b_{1}(r)=\min \left(a_{1}(r), a_{2}(r)\right)$ and let $b_{2}(r)=\max \left(a_{1}(r), a_{2}(r)\right)$. For $r<0$, let $b_{1}(r)=\min \left(0, a_{1}(r)\right)$ and let $b_{2}(r)=\max \left(0, a_{1}(r)\right)$. Then for $x>0, y>0, x \neq y$,

$$
M_{2, b_{1}(r)} \leq L_{r}(x, y) \leq M_{2, b_{2}(r)},
$$

with the choices $b_{1}(r), b_{2}(r)$ best possible. 
We note here that Stolarsky [15] also showed $L_{r}(x, y) \leq M_{2,(r+1) / 3}$ for $-1 \leq r \leq 1 / 2$ or $r \geq 2$ and $L_{r}(x, y) \geq M_{2,(r+1) / 3}$ for $1 / 2 \leq r \leq 2$ or $r \leq-1$. Our next result gives an extension of [7, Theorem 3.1] for in the case $n=2, q_{1}=q_{2}=1 / 2$. We leave the proof to the interested reader by pointing out that one can prove it by using similar approaches as in the proof of Theorem 2.1.

Theorem 4.2. Let $n=2, q_{1}=q_{2}=1 / 2$. For $1 / 2 \leq s<1$,

$$
C_{1, s, 0}\left(\frac{1}{2}\right) \geq \Delta_{1, s, 0} \geq \frac{1}{1-s} .
$$

Equality holds for the left-hand side inequality of (4.2) if and only if $x_{1}=0$ or $s=1 / 2$ (note that it is assumed that $x_{1}<x_{2}$ here). Equality holds for the right-hand side inequality of (4.2) if and only if $s=1 / 2$. The above inequalities are reversed when $0<s \leq 1 / 2$ with similar conditions for equalities. Moreover, for $r>1$,

$$
C_{r, 1,0}\left(\frac{1}{2}\right) \geq \Delta_{r, 1,0}>\frac{r}{r-1},
$$

with equality holding for the above left-hand side inequality if and only if $x_{1}=0$.

We note here that the lower bound in (4.2) (and the corresponding upper bound when $0<s \leq 1 / 2)$ is due to Bloom and Seiffert [3], who also mentioned that the inequality $\left(G_{2}+\right.$ $\left.2 A_{2}\right) / 3 \leq M_{2,2 / 3}$ was proposed at the " 16 th Austrian-Polish Mathematics Competition 1993." Stolarsky [15] has shown that $M_{2,2 / 3}<I$, it then follows that $\left(G_{2}+2 A_{2}\right) / 3<I$, a result of Sándor [11]. One can obtain similar results by using Theorems 4.1 and 4.2 via this approach. Before we state our next result, we need a lemma.

LeMma 4.3. For $t \geq 0, s \geq 1 / 2$,

$$
\left(\frac{t^{s}-1}{t-1}\right)^{1 /(s-1)} \geq\left(1+t^{s}\right)^{1 / s}
$$

with the above inequality reversed when $0<s \leq 1 / 2$.

Proof. We will prove the case $1 / 2<s<1$ and the proofs for the other cases are similar. By homogeneity, we may also assume that $0 \leq t \leq 1$. Define

$$
f(t)=\left(\frac{t^{s}-1}{t-1}\right)^{1 /(s-1)}\left(1+t^{s}\right)^{-1 / s}
$$

and it suffices to show $f(t) \geq 1$. Note that

$$
f^{\prime}(t)=\left(\frac{t^{s}-1}{t-1}\right)^{(2-s) /(s-1)}\left(1+t^{s}\right)^{-(1+s) / s}\left(\frac{t^{2 s-1}-(2 s-1) t^{s}+(2 s-1) t^{s-1}-1}{(1-s)(t-1)^{2}}\right) .
$$

Let

$$
g(t)=t^{2 s-1}-(2 s-1) t^{s}+(2 s-1) t^{s-1}-1 .
$$


Then

$$
g^{\prime}(t)=(2 s-1) t^{s-2}\left(t^{s}-s t+(s-1)\right) \leq 0 .
$$

Hence $g(t) \geq g(1)=0$ and it follows that $f^{\prime}(t) \geq 0$ so that $f(t) \geq f(0)=1$ which completes the proof.

Corollary 4.4. For $x, y>0, x \neq y$,

$$
\frac{2}{e} L_{2}(x, y)<I(x, y)<\frac{2}{e} L_{2}(x, y)+\left(1-\frac{2}{e}\right) L_{-1}(x, y) .
$$

Proof. The first inequality with "<" replaced by " $\leq$ " follows from Lemma 4.3 by replacing $t$ with $x / y$ and letting $s \rightarrow 1^{-}$. The fact that a strict inequality holds here is due to Sándor [12]. The second inequality is due to Alzer and Qiu [2, Theorem 1], it follows from the inequality $I(x, y) \leq M_{2, \log 2}$ by Theorem 4.1 and the inequality $M_{2, \log 2}<2 / e L_{2}(x, y)+(1-$ $(2 / e)) L_{-1}(x, y)$ by Theorem 4.2 with $s=\log 2$.

Corollary 4.5. For $x>0, y>0, x \neq y$, and $0<r<1 / 2$,

$$
\left(1-\frac{2}{r^{1 /(r-1)}}\right) L_{-1}(x, y)+\frac{2}{r^{1 /(r-1)}} L_{2}(x, y)<L_{r}(x, y)<\frac{(2-r) L_{-1}(x, y)+(1+r) L_{2}(x, y)}{3},
$$

with the above inequality reversed when $1 / 2<r \leq 2, r \neq 1$.

We point out here that since $r \mapsto L_{r}(x, y), x \neq y$ is strictly increasing (see [14]), one has $L_{r} \leq L_{2}=A_{2}$ for $0 \leq r \leq 2$ and it follows from Theorem 4.1 that $(r-1) \log 2 / \log r \leq 1$ for $1 / 2 \leq r \leq 2, r \neq 1$ ( since $L_{r} \leq A_{2}$ and $L_{r} \leq M_{2,(r-1) \log 2 / \log r}$ which is the best possible). Hence one can apply Theorems 4.1 and 4.2 to prove Corollary 4.5.

Note here that the limit case of $r \rightarrow 0^{+}$for the right-hand side inequality of (4.10) gives a result of Carlson [4]. Similarly, the limit case of $r \rightarrow 1$ for the corresponding inequality gives a result of Sándor [11].

\section{Acknowledgments}

This work was partially carried out while the author was visiting the American Institute of Mathematics. We would like to thank both the American Institute of Mathematics and the Centre de Recherches Mathématiques at the Université de Montréal for their generous support and hospitality.

\section{References}

[1] H. Alzer, The inequality of Ky Fan and related results, Acta Applicandae Mathematicae 38 (1995), no. 3, 305-354.

[2] H. Alzer and S.-L. Qiu, Inequalities for means in two variables, Archiv der Mathematik 80 (2003), no. 2, 201-215.

[3] D. M. Bloom and H.-J. Seiffert, Problems and solutions: solutions: 10289, The American Mathematical Monthly 103 (1996), no. 8, 696-697. 


\section{On an inequality of Diananda. Part III}

[4] B. C. Carlson, The logarithmic mean, The American Mathematical Monthly 79 (1972), no. 6, 615-618.

[5] J. Chen and Z. Wang, A reverse of an analytic inequality, Journal of Ningbo University Science Edition (1992), no. 2, 12-14 (Chinese).

[6] __ A proof to an analytic inequality, Journal of Ningbo University Science Edition (1994), no. 1, 13-15 (Chinese).

[7] P. Gao, On an inequality of Diananda, International Journal of Mathematics and Mathematical Sciences 2003 (2003), no. 32, 2061-2068.

[8] _ On an inequality of Diananda. II, International Journal of Mathematics and Mathematical Sciences 2005 (2005), no. 9, 1381-1386.

[9] Z. Luo, L. Lai, and J. J. Wen, The dimension-descending computation method of global optimizations for symmetric functions, Journal of Chengdu University. Natural Sciences 21 (2002), no. 1, 5-11 (Chinese).

[10] A. O. Pittenger, Inequalities between arithmetic and logarithmic means, Univerzitet u Beogradu. Publikacije Elektrotehničkog Fakulteta. Serija Matematika i Fizika (1980), no. 678-715, 15-18 (1981).

[11] J. Sándor, A note on some inequalities for means, Archiv der Mathematik 56 (1991), no. 5, 471473.

[12] On certain inequalities for means, Journal of Mathematical Analysis and Applications 189 (1995), no. 2, 602-606.

[13] D. Shen, On the coefficients and zeros of a polynomial, Journal of Approximation Theory 96 (1999), no. 2, 405-410.

[14] K. B. Stolarsky, Generalizations of the logarithmic mean, Mathematics Magazine 48 (1975), 8792.

[15] _ The power and generalized logarithmic means, The American Mathematical Monthly 87 (1980), no. 7, 545-548.

[16] S. Wu, Generalization and sharpness of the power means inequality and their applications, Journal of Mathematical Analysis and Applications 312 (2005), no. 2, 637-652.

[17] $\mathrm{C} . \mathrm{Wu}, \mathrm{W}$. Wang, and $\mathrm{L} . \mathrm{Fu}$, Inequalities for symmetric functions and their applications, Journal of Chengdu University of Science and Technology (1982), no. 1, 103-108 (Chinese).

Peng Gao: Centre de Recherches Mathématiques, Université de Montréal, BP 6128,

Centre-Ville Station, Montréal, QC, Canada H3C 3J7

Current address: Department of Computer and Mathematical Sciences, University of Toronto at Scarborough, 1265 Military Trail, Toronto, ON, Canada M1C 1A4

E-mail addresses: gao@crm.umontreal.ca; penggao@utsc.utoronto.ca 


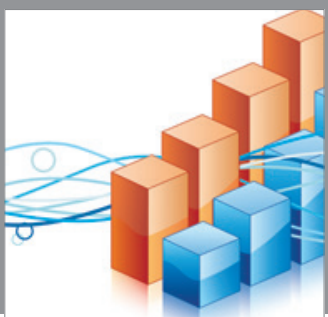

Advances in

Operations Research

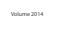

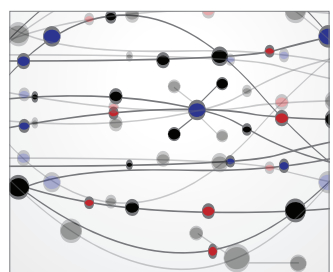

\section{The Scientific} World Journal
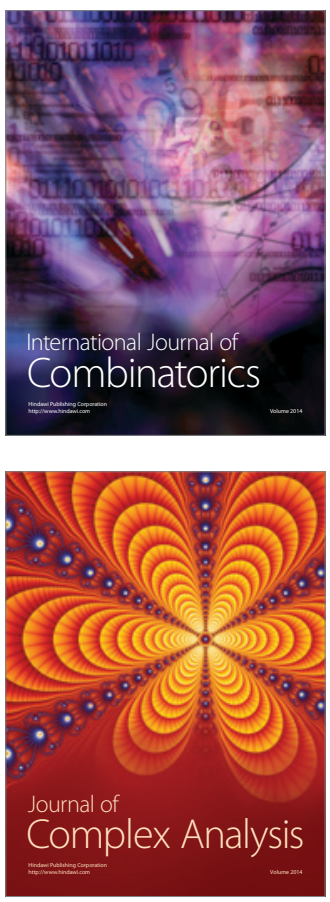

International Journal of

Mathematics and

Mathematical

Sciences
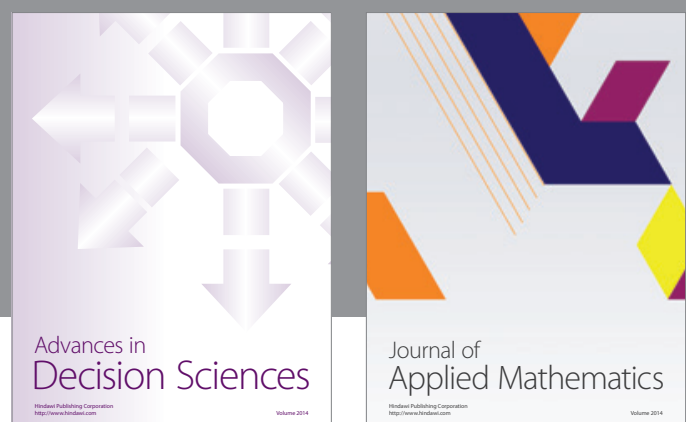

Journal of

Applied Mathematics
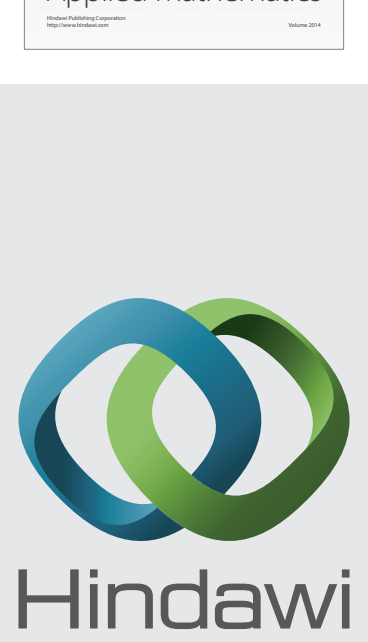

Submit your manuscripts at http://www.hindawi.com
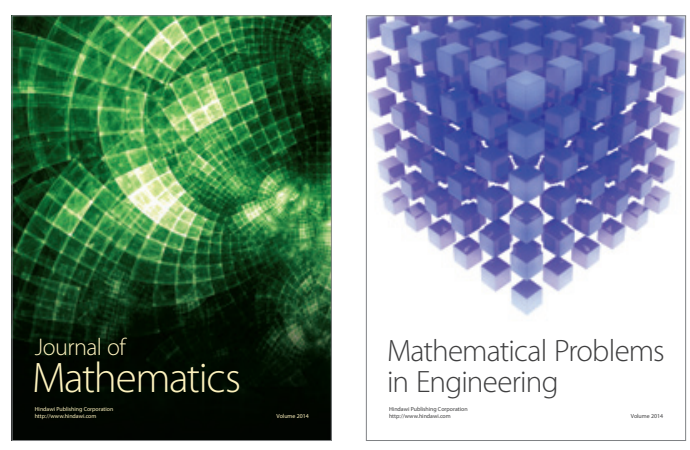

Mathematical Problems in Engineering
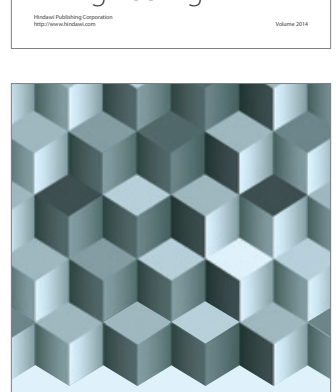

Journal of

Function Spaces
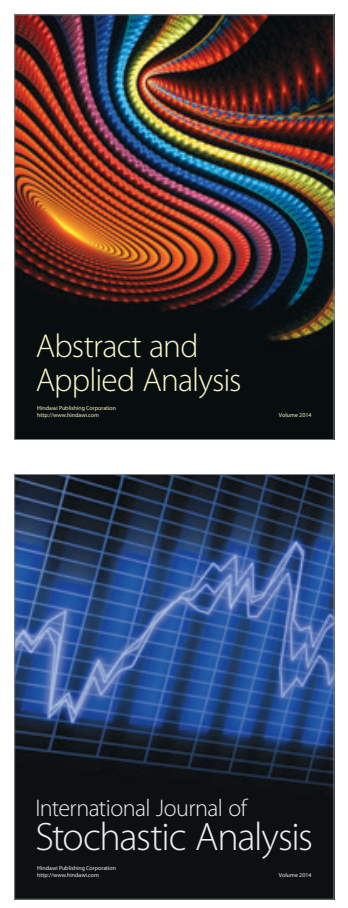

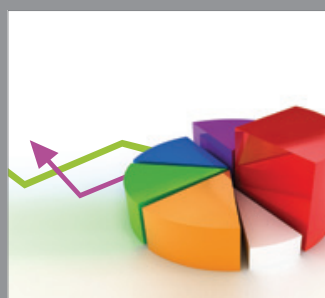

ournal of

Probability and Statistics

Promensencen
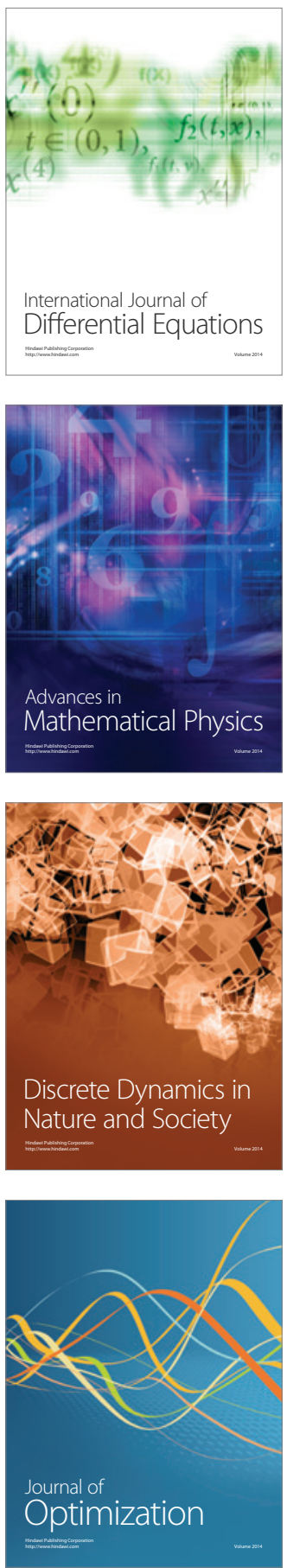